\title{
Are Portable Stereophotogrammetric Devices Reliable in Facial Imaging? A Validation Study of VECTRA H1 Device
}

\author{
Daniele Gibelli, MD, PhD, ${ }^{*}$ Valentina Pucciarelli, MSBiotech, PhD, $\dagger$ \\ Annalisa Cappella, PhD, $\downarrow$ Claudia Dolci, MD, PhD, $\S$ and Chiarella Sforza, MD, PhD, //
}

Purpose: Modern 3-dimensional (3D) image acquisition systems represent a crucial technologic development in facial anatomy because of their accuracy and precision. The recently introduced portable devices can improve facial databases by increasing the number of applications. In the present study, the VECTRA $\mathrm{H1}$ portable stereophotogrammetric device was validated to verify its applicability to 3D facial analysis.

Materials and Methods: Fifty volunteers underwent 4 facial scans using portable VECTRA H1 and static VECTRA M3 devices ( 2 for each instrument). Repeatability of linear, angular, surface area, and volume measurements was verified within the device and between devices using the Bland-Altman test and the calculation of absolute and relative technical errors of measurement (TEM and rTEM, respectively). In addition, the 2 scans obtained by the same device and the 2 scans obtained by different devices were registered and superimposed to calculate the root mean square (RMS; point-to-point) distance between the 2 surfaces.

Results: Most linear, angular, and surface area measurements had high repeatability in M3 versus M3, H1 versus $\mathrm{H} 1$, and $\mathrm{M} 3$ versus $\mathrm{H} 1$ comparisons (range, 82.2 to $98.7 \%$; TEM range, 0.3 to $2.0 \mathrm{~mm}, 0.4^{\circ}$ to $1.8^{\circ}$; rTEM range, 0.2 to $3.1 \%$ ). In contrast, volumes and RMS distances showed evident differences in M3 versus M3 and H1 versus H1 comparisons and reached the maximum when scans from the 2 different devices were compared.

Conclusion: The portable VECTRA H1 device proved reliable for assessing linear measurements, angles, and surface areas; conversely, the influence of involuntary facial movements on volumes and RMS distances was more important compared with the static device.

(C) 2018 American Association of Oral and Maxillofacial Surgeons

J Oral Maxillofac Surg 76:1772-1784, 2018

In recent decades, new 3-dimensional (3D) image acquisition systems have revolutionized procedures for assessing facial morphology and metrics. As a consequence, traditional methods of direct anthropometry (based on the use of calipers) were replaced by innovative methods of $3 \mathrm{D}$ analysis, ${ }^{1}$ which allow not only linear and angular, but also surface areas and volume measurements,
Received from Laboratorio di Anatomia Funzionale dell'Apparato Stomatognatico (LAFAS), Dipartimento di Scienze Biomediche per la Salute, Università degli Studi di Milano, Milan, Italy.

*Assistant Professor.

$\dagger$ Resident.

$\ddagger$ Resident.

$\S$ Assistant Professor.

||Professor

Daniele Gibelli and Valentina Pucciarelli equally contributed to this work.

Conflict of Interest Disclosures: The VECTRA H1 system was kindly provided by Canfield Scientific, Inc (Fairfield, NJ). All data acquisition and analyses were performed by the authors independently of Canfield Scientific. Dr Sforza received a free and unconditional temporary loan of the VECTRA H1 system to be used exclusively for the present investigation. The other authors have no relevant financial relationship(s) with a commercial interest.

Address correspondence and reprint requests to Prof Sforza: Dipartimento di Scienze Biomediche per la Salute, Università degli Studi di Milano, via Mangiagalli 31, I-20133 Milan, Italy; e-mail: chiarella.sforza@unimi.it

Received November 262017

Accepted January 172018

(c) 2018 American Association of Oral and Maxillofacial Surgeons

0278-2391/18/30087-9

https://doi.org/10.1016/i.joms.2018.01.021 
and registration and superimposition of 3D surfaces. ${ }^{2-5}$

One of the most frequently applied technologies for the assessment of facial soft tissues is stereophotogrammetry, which consists of at least 2 cameras that capture the same image simultaneously from different angles. ${ }^{6}$ Then, the different images acquired with overlapping fields of view are merged into a 3D model. This type of technology allows quick image acquisition, thus avoiding the effects of involuntary head movements and mimicry, and does not require contact with the facial surface, which can increase errors because of traditional methods of measurement. ${ }^{1,7,8}$

Stereophotogrammetry is currently applied in different research fields linked to facial anatomy, maxillofacial and esthetic surgery, assessment of facial modifications during growth in children, ${ }^{9}$ analysis of facial features in patients affected by acquired and genetic pathologies, ${ }^{10-12}$ evaluation of anatomic standards of symmetry, ${ }^{13}$ the study of mimicry in healthy people, and patients affected by facial impairments. ${ }^{13-16}$

However, current stereophotogrammetric systems have some limits, with the most important being the high encumbrance of the device, including the entire system with multiple cameras. As a consequence, these instruments cannot be easily moved to other locations, and their transport is discouraged. In addition, stereophotogrammetric devices are expensive and require frequent calibration. ${ }^{17}$ These limits represent a serious obstacle for the acquisition of facial images and the construction of a complete database, especially for what concerns the analysis of patients affected by severe pathologies or genetic syndromes, patients who cannot move independently, or hospitalized patients.

In recent years novel models of portable stereophotogrammetric devices have appeared on the market; these systems can obtain a 3D facial model through compact instruments with cameras and a laptop. ${ }^{18}$ In contrast to traditional static systems, these instruments require the operator to acquire 3 images of the same subject from different angles within a limited period to obtain a final 3D facial model. These new devices could provide a strong innovation to the study of facial anatomy and could help enlarge facial databases, thus extending fields of possible research.

However, the hand-held acquisition systems need to be validated to verify that they can provide a reliable 3D acquisition of faces. From this point of view, their main weak point is the need for a sequential acquisition of 3 images, whereas the static stereophotogrammetric systems acquire all images at the same time. This difference can increase the possible influence of involuntary head and facial movements in the reconstruction of the final 3D model. ${ }^{18}$
Thus far, only 1 article has performed a validation of a portable stereophotogrammetric device (VECTRA H1; Canfield Scientific, Inc, Fairfield, NJ) in comparison with a 3dMD static system (3dMD LLC, Atlanta, GA) ${ }^{18}$ The investigators analyzed the repeatability of linear measurements from the same individuals taken from 3D facial models obtained through the portable and static devices. In addition, a superimposition procedure was performed registering the VECTRA H1 facial model onto the $3 \mathrm{dMD}$ model. ${ }^{18}$

Although the study provides an important contribution in verifying the reliability of the VECTRA H1 system for assessing facial morphology, also confirming the important influence of involuntary movements, some aspects of validation were not fully addressed. For example, intra-device repeatability still needs to be verified by analyzing 2 scans obtained through the same device and comparing the performances of the 2 systems. In addition, surface areas and volumes were not used to validate the novel portable systems, although they represent important measurements in 3D facial assessment.

The present study aimed at extending the existing literature concerning the validation of portable stereophotogrammetric systems applied to facial anatomy. In particular, the VECTRA H1 system, already tested by Camison et al, ${ }^{18}$ was compared with the static device marketed by the same company, the VECTRA M3, providing further data for the validation of this promising and innovative technology.

\section{Materials and Methods}

\section{SAMPLE RECRUITMENT}

Fifty volunteers (16 men and 34 women; 19 to $61 \mathrm{yr}$ old) were recruited for the present study. The sample size was automatically determined according to the database of subjects who underwent scanning with the M3 and H1 systems (confidence level, 95\%; confidence interval, 5\%). Exclusion criteria were deformations, pathologies, impairments, or traumatic events involving the facial area. Volunteers with beards were excluded from the study, because the stereophotogrammetric device cannot acquire surface areas covered by excess facial hair. All participants signed an informed consent. The study was performed in accord with guidelines provided by the Declaration of Helsinki and was approved by the university ethical committee (26.03.14; number 92/14).

\section{D ACQUISITION}

Each volunteer was requested to remove any jewelry and hair was pulled back through a band to expose the forehead and ears. A series of 50 landmarks was marked on each face using eyeliner 
according to the authors' standardized procedure for $3 \mathrm{D}$ acquisition. ${ }^{6}$

Three calibrated operators performed all scans; their experience with the static system ranged from 4 to 6 years and their experience with the portable instrument was 3 months.

An image of each participant was acquired twice using the portable VECTRA H1 system and twice using the static VECTRA M3 device (Fig 1). The acquisitions were performed consecutively in the same room. A few minutes elapsed between the scans made with the 2 devices.

Modalities of acquisition differed according to the type of device; for these 2 devices the volunteers were requested to sit on a stool and maintain a neutral position. For the static VECTRA M3, the stool was in front of the 3-pod system; for the portable VECTRA $\mathrm{H} 1$, the stool was in a corner of the room. The operator
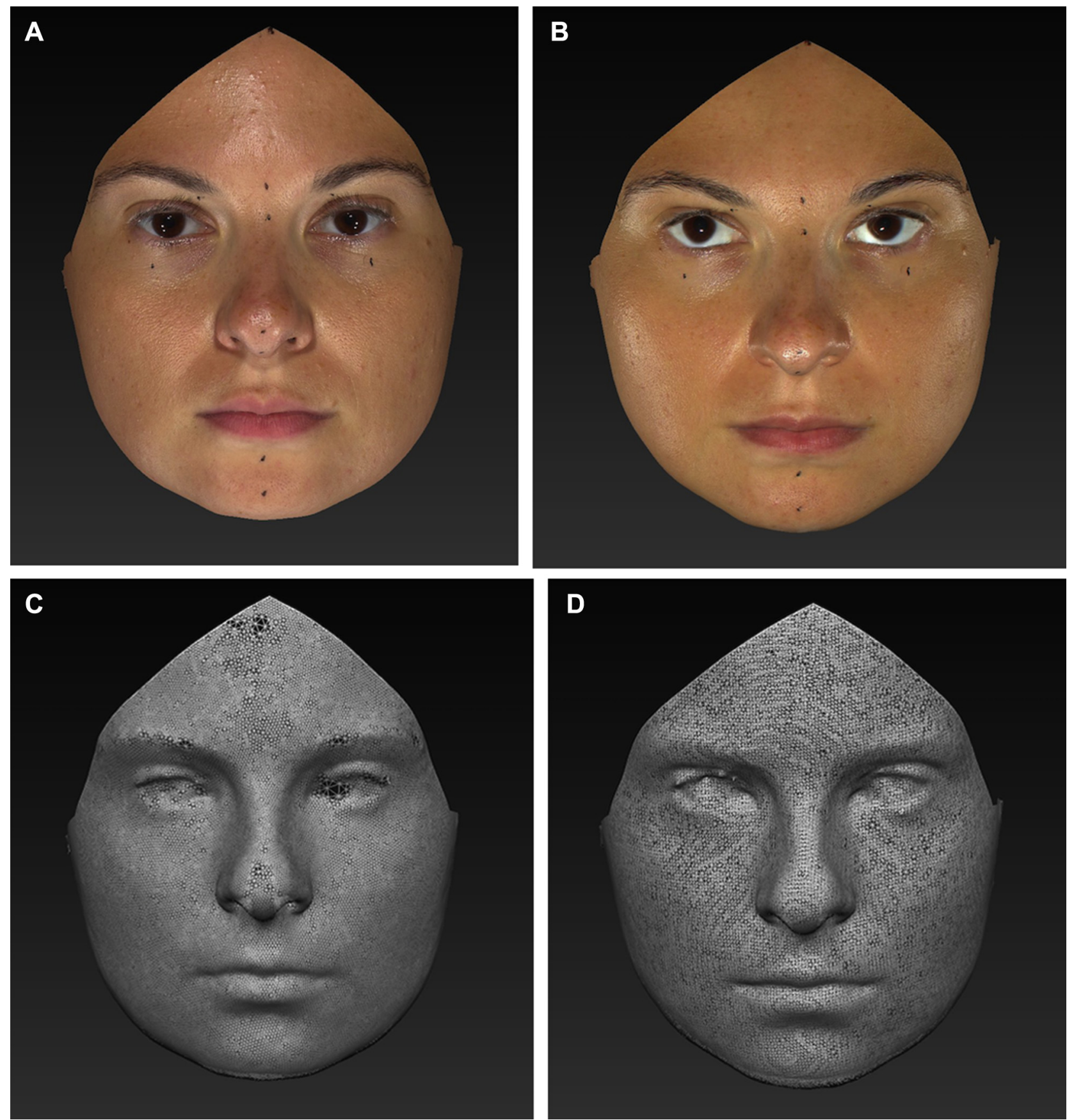

FIGURE 1. Examples of facial scans obtained with the static $M 3$ and portable $H 1$ VECTRA systems. $A, M 3$ scan. $B$, Scan of the same subject with the H1. C, M3 model without texture. $D, \mathrm{H} 1$ model without texture. The subject is one of the authors. 
took the 3 images some seconds apart in the specific conditions suggested by the manufacturer: the first capture occurred while keeping the camera $45^{\circ}$ to the volunteer's right side and approximately 20 to $30 \mathrm{~cm}$ below the volunteer's face, the second capture occurred in the frontal position, and the third capture occurred with the camera $45^{\circ}$ to the volunteer's left side, in a similar condition as the first capture. All procedures were performed according to the manufacturer's guidelines. During the entire acquisition session, the device was linked to a laptop computer to verify the accuracy of $3 \mathrm{D}$ reconstructions.

The same procedure of acquisition was applied 5 times to a mannequin head, for 10 scans using the VECTRA H1 and 10 scans using the VECTRA M3; this tested the same measurements of 3D-to-3D surface registration in the absence of head and facial movements.

\section{DATA ELABORATION}

Each 3D facial model was elaborated by VAM elaboration software (Canfield Scientific, Inc). Analysis was performed for 4 kinds of measurements: linear measurements and angles, surface areas, volumes, and 3D surface registration and superimposition.

Fifteen linear measurements and 12 angles were automatically calculated through Faces software, developed by the authors' laboratory specifically for the extraction of metric measurements from coordinates (Tables 1,2), after the selection on the 3D

\section{Table 1. LIST OF ABBREVIATIONS AND DEFINITIONS} FOR ANALYZED LINEAR DISTANCES

\begin{tabular}{|c|c|}
\hline Abbreviation & Definition \\
\hline \multicolumn{2}{|l|}{ Frontal plane } \\
\hline $\operatorname{tr}-n$ & Length of forehead \\
\hline n-pg & Total facial height \\
\hline n-sn & Nasal height \\
\hline sn-pg & Lower facial height \\
\hline \multicolumn{2}{|c|}{ Horizontal plane } \\
\hline$e x_{r}-e x_{1}$ & Intercanthal distance \\
\hline $\mathrm{zy}_{\mathrm{r}}-\mathrm{zy}_{1}$ & Facial width \\
\hline $\mathrm{t}_{\mathrm{r}}-\mathrm{t}_{1}$ & Middle facial width \\
\hline $\mathrm{ch}_{\mathrm{r}}-\mathrm{ch}_{1}$ & Mouth width \\
\hline $\mathrm{cph}_{\mathrm{r}}-\mathrm{cph}_{1}$ & Philtrum breadth \\
\hline $\mathrm{go}_{\mathrm{r}}-\mathrm{go}_{1}$ & Lower facial width \\
\hline \multicolumn{2}{|l|}{ Sagittal plane } \\
\hline$t_{m}-n$ & Upper facial depth \\
\hline$t_{m}-s n$ & Midfacial depth \\
\hline$t_{m}-p g$ & Lower facial depth \\
\hline pg-go $\mathrm{m}$ & Mandibular body length \\
\hline $\mathrm{t}_{\mathrm{m}}-\mathrm{go}_{\mathrm{m}}$ & Mandibular ramus length \\
\hline
\end{tabular}

Abbreviations: 1, left; $\mathrm{m}$, mid-landmark; $r$, right.

Gibelli et al. Validation of Portable Stereophotogrammetry. J Oral Maxillofac Surg 2018.

\begin{tabular}{|c|c|}
\hline Abbreviation & Definition \\
\hline \multicolumn{2}{|l|}{ Frontal plane } \\
\hline $\mathrm{en}_{\mathrm{r}}-\mathrm{ex}$ vs TH & $\begin{array}{l}\text { Inclination of right palpebral } \\
\text { fissure vs true horizontal plane }\end{array}$ \\
\hline $\mathrm{en}_{1}-\mathrm{ex}_{1}$ vs TH & $\begin{array}{l}\text { Inclination of left palpebral fissure } \\
\text { vs true horizontal plane }\end{array}$ \\
\hline \multicolumn{2}{|l|}{ Horizontal plane } \\
\hline$t_{r}-n-t_{1}$ & Upper facial convexity \\
\hline$t_{r}-$ prn- $t_{1}$ & Middle facial convexity \\
\hline$t_{r}-p g-t_{1}$ & Lower facial convexity \\
\hline go $_{\mathbf{r}}-\mathrm{pg}-\mathrm{go}_{1}$ & Mandibular convexity \\
\hline \multicolumn{2}{|l|}{ Sagittal plane } \\
\hline n-sn-pg & Facial convexity (excluding nose) \\
\hline n-prn-pg & Facial convexity (including nose) \\
\hline sn-n-prn & Nasal convexity \\
\hline $\mathrm{t}_{\mathrm{r}}-\mathrm{go}_{\mathrm{r}}-\mathrm{pg}$ & Right gonial angle \\
\hline $\mathrm{t}_{1}-\mathrm{go}_{1}-\mathrm{pg}$ & Left gonial angle \\
\hline$\left(t_{m}-n\right)$ vs $\left(g o_{m}-p g\right)$ & $\begin{array}{l}\text { Facial divergence (midfacial to } \\
\text { mandibular plane angle) }\end{array}$ \\
\hline
\end{tabular}

Abbreviations: 1, left; m, mid-landmark; r, right; TH, true horizontal plane.

Gibelli et al. Validation of Portable Stereophotogrammetry. J Oral Maxillofac Surg 2018.

model of 12 facial landmarks previously marked on the volunteers' faces and defined according to Farkas $^{19}$ (Fig 2). Linear distances and angles were chosen to provide a general evaluation of facial metric characteristics according to all anatomic planes. ${ }^{11}$

For the assessment of reliability of surface area and volume measurements, a facial area of interest (FAI) was selected from the 3D models as the facial surface within the trichion, frontotemporale, zygion, tragion, gonion, and gnathion landmarks. Selection of the FAI was performed automatically by the $3 \mathrm{D}$ elaboration software once the eyeliner markers were identified on the digital reconstruction. The entire procedure has been published and was found to be well repeatable. $^{20}$ The surface area and volume of each FAI were automatically calculated by VAM software.

The FAIs belonging to the same participant were registered and superimposed one on the other to assess the point-to-point root mean square (RMS) distance between the 2 3D surfaces. In detail, the 2 scans obtained using the same device (VECTRA H1 or VECTRA M3) were registered and then superimposed (Fig 2). Then, the first scan obtained with the VECTRA H1 was superimposed onto the first scan obtained with the VECTRA M3. Registration was automatically performed by VAM software according to the shortest point-to-point distance between the 2 3D surfaces. Then, mean and RMS point-to-point distances were automatically calculated. 


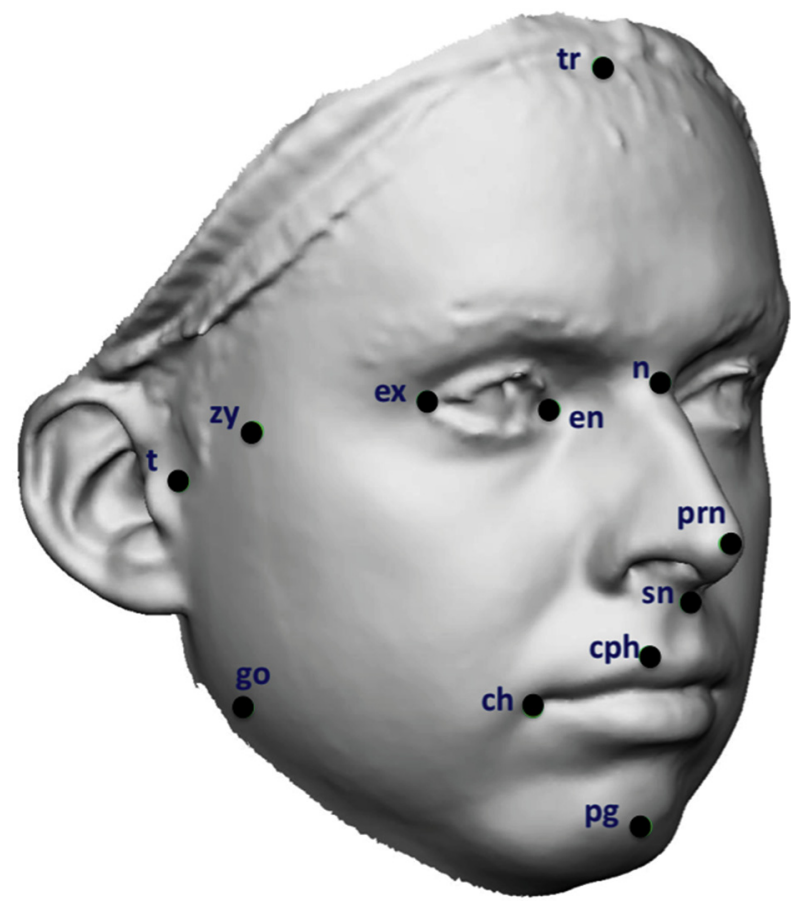

FIGURE 2. Detail of 12 facial landmarks used for the automatic calculation of distances and angles. ${ }^{18}$ The subject is one of the authors. ch, cheilion; cph, crista philtri; en, endocanthion; ex, exocanthion; go, gonion; n, nasion; pg, pogonion; prn, pronasale; sn, subnasale; $\mathrm{t}$, tragion; tr, trichion; zy, zygion.

Gibelli et al. Validation of Portable Stereophotogrammetry. J Oral Maxillofac Surg 2018.

In addition, all described registration and superimposition procedures were applied to the scans of the mannequin head.

\section{STATISTICAL ANALYSIS}

Intra-device repeatability of linear measurements, angles, surface area, and volume of FAIs was assessed using the Bland-Altman test. ${ }^{21}$ In addition, the absolute (distance in millimeters, angles in degrees, surface in square centimeters, and volume in cubic centimeters) and relative (percentage) technical errors of measurement (TEM and rTEM, respectively), expressing the error magnitude relative to the size of measurements, were calculated. ${ }^{22}$ The same analyses were performed comparing the metric parameters taken from the first scan using the VECTRA H1 and the first scan using the VECTRA M3 and between the mean of the measurements from the 2 VECTRA H1 models and from the 2 VECTRA M3 facial models. The rTEM values were evaluated according to the scale proposed by Camison et $\mathrm{al}^{18}$ who classified 5 categories (excellent, <1\%; very good, 1 to $3.9 \%$; good, 4 to $6.9 \%$; moderate, 7 to 9.9\%; poor, $>10 \%$ poor).

For RMS point-to-point distance, differences in values obtained from the registration of scans performed using the same device and between the first
$\mathrm{H} 1$ and the first M3 scans were assessed by 1-way analysis of variance (ANOVA; $P<.05$ ). Post hoc tests were performed using the Student $t$ test after correcting for degrees of freedom. The same procedure and test were applied to RMS values obtained from the mannequin head scans.

\section{Results}

Results for linear measurements and angles are presented in Tables 3 to 5 . The repeatability of most linear measurements and angles ranged from 82.2 to $98.7 \%$ (TEM range, 0.3 to $2.0 \mathrm{~mm}, 0.4^{\circ}$ to $1.8^{\circ}$; rTEM range, 0.2 to $3.1 \%$ ) in different groups of comparisons, except for the labial and periocular regions. The distance from crista philtri to crista philtri (cph-cph) showed the worst repeatability of 30.3 to $62.3 \%$ (TEM, 0.8 to $1.4 \mathrm{~mm}$; rTEM, 6.7 to $12.4 \%$ ), whereas the inclinations of the 2 palpebral fissures versus the true horizontal plane had a repeatability of 49.9 to $61.7 \%$ (TEM, $0.9^{\circ}$ to $1.8^{\circ}$; rTEM, 6.7 to $14.9 \%$ ). For all other evaluated metric measurements, the rTEM was mainly classified as excellent for intra-device (M3 vs M3 and H1 vs H1) comparisons compared with very good for M3 versus H1 comparisons (first assessment and mean; Table 5; represented in Fig 3 by different gray scales, with a lighter shade of the linear distances and angles indicating superior repeatability).

In general, repeatability and TEM and rTEM values for linear distances and angles worsened for $\mathrm{M} 3$ versus M3 and $\mathrm{H} 1$ versus $\mathrm{H} 1$ comparisons and reached the minimum when scans from the 2 different devices were compared. The application of the same analyses to the mean of measurements from the 2 scans performed using the $\mathrm{H} 1$ and $\mathrm{M} 3$ slightly improved the repeatability and TEM and rTEM values for most measurements. Classification of rTEM by the comparison of mean values assessed for the $\mathrm{H} 1$ and $\mathrm{M} 3$ did not vary for most measurements.

Repeatability of FAI surface area and volume between 2 scans obtained with the VECTRA M3 were high, with excellent and very good rTEMs, respectively. Performance of the VECTRA H1 was the same for FAI surface area but lower for the relevant volume, with a moderate rTEM. The comparison between the first scan with the VECTRA H1 and the first scan with the VECTRA M3 confirmed the lower reliability in assessing volumes compared with surface areas. The comparison of mean values between the 2 scans acquired through the same device slightly ameliorated the performances. For surface area, a similar trend was observed for TEM values: inter-instrument comparisons yielded values approximately twice as large as intra-instrument assessments. In contrast, volume assessments had important TEM values for M3 versus $\mathrm{H} 1$ and $\mathrm{H} 1$ versus $\mathrm{H} 1$ comparisons; acceptable 
Table 3. REPEATABILITY ACCORDING TO BLAND-ALTMAN TEST FOR LINEAR DISTANCES AND ANGLES

\begin{tabular}{|c|c|c|c|c|}
\hline & M3 vs M3 & H1 vs $\mathrm{H} 1$ & M3 vs H1 & M3 vs H1 (Mean) \\
\hline \multicolumn{5}{|l|}{ Linear distances } \\
\hline \multicolumn{5}{|l|}{ Frontal plane } \\
\hline $\operatorname{tr}-n$ & 96.0 & 91.0 & 90.0 & 92.1 \\
\hline n-pg & 97.1 & 96.7 & 97.0 & 91.6 \\
\hline n-sn & 97.1 & 94.9 & 90.8 & 94.1 \\
\hline sn-pg & 96.3 & 94.6 & 91.0 & 93.5 \\
\hline \multicolumn{5}{|l|}{ Horizontal plane } \\
\hline $\mathrm{ex}_{\mathrm{r}}-\mathrm{ex} \mathrm{x}_{1}$ & 95.3 & 95.0 & 94.5 & 94.9 \\
\hline $\mathrm{zy}_{\mathrm{r}}-\mathrm{zy}_{1}$ & 98.7 & 97.7 & 97.1 & 96.7 \\
\hline$t_{r}-t_{1}$ & 97.1 & 94.8 & 93.7 & 95.4 \\
\hline $\mathrm{ch}_{\mathrm{r}}-\mathrm{ch}_{1}$ & 90.5 & 87.3 & 86.2 & 88.6 \\
\hline $\mathrm{cph}_{\mathrm{r}}-\mathrm{cph} \mathrm{h}_{1}$ & 62.3 & 47.9 & 30.3 & 39.2 \\
\hline $\mathrm{go}_{\mathrm{r}}-\mathrm{go}_{1}$ & 96.5 & 94.5 & 92.1 & 92.3 \\
\hline \multicolumn{5}{|l|}{ Sagittal plane } \\
\hline$t_{m}-n$ & 94.3 & 96.0 & 92.9 & 98.0 \\
\hline$t_{m}-s n$ & 94.9 & 94.4 & 91.6 & 92.8 \\
\hline$t_{m}-p g$ & 95.0 & 96.6 & 93.3 & 92.6 \\
\hline pg-go & 96.7 & 92.5 & 92.0 & 92.8 \\
\hline$t_{m}-g o_{m}$ & 90.0 & 93.5 & 90.4 & 87.5 \\
\hline \multicolumn{5}{|l|}{ Angles } \\
\hline \multicolumn{5}{|l|}{ Frontal plane } \\
\hline $\mathrm{en}_{\mathrm{r}}-\mathrm{ex} \mathrm{x}_{\mathrm{r}}$ vs TH & 53.3 & 59.1 & 58.1 & 50.4 \\
\hline $\mathrm{en}_{1}-\mathrm{ex}_{1}$ vs TH & 59.0 & 61.7 & 58.6 & 49.9 \\
\hline \multicolumn{5}{|l|}{ Horizontal plane } \\
\hline$t_{r}-n-t_{1}$ & 96.6 & 92.5 & 93.7 & 94.9 \\
\hline$t_{r}-$ prn-t $t_{1}$ & 96.1 & 94.1 & 93.6 & 95.0 \\
\hline$t_{r}-p g-t_{1}$ & 92.2 & 95.3 & 91.9 & 93.9 \\
\hline go $_{\mathbf{r}}-\mathrm{pg}_{-} \mathrm{go}_{1}$ & 93.3 & 93.1 & 89.5 & 87.7 \\
\hline \multicolumn{5}{|l|}{ Sagittal plane } \\
\hline n-sn-pg & 98.4 & 97.1 & 96.9 & 97.7 \\
\hline n-prn-pg & 97.2 & 98.4 & 96.5 & 97.8 \\
\hline sn-n-prn & 90.0 & 88.9 & 82.2 & 85.3 \\
\hline $\mathrm{t}_{\mathrm{r}}-\mathrm{go}_{\mathrm{r}}-\mathrm{pg}$ & 92.9 & 94.7 & 92.9 & 96.1 \\
\hline $\mathrm{t}_{1}-\mathrm{go}_{\mathrm{l}}-\mathrm{pg}$ & 95.0 & 94.8 & 92.3 & 92.6 \\
\hline$\left(t_{m}-n\right)$ vs $\left(g_{m}-p g\right)$ & 96.2 & 94.7 & 92.2 & 92.6 \\
\hline
\end{tabular}

Note: All values are percentages.

Abbreviations: See Tables 1 and 2.

Gibelli et al. Validation of Portable Stereophotogrammetry. J Oral Maxillofac Surg 2018.

differences were found only for M3 versus M3 comparisons (Table 6).

For surface registration (Fig 4), the RMS was lower in the comparison of scans obtained with the VECTRA M3 (average, $0.22 \mathrm{~mm}$; standard deviation [SD], $0.14 \mathrm{~mm}$ ) than of $3 \mathrm{D}$ facial models acquired with the VECTRA H1 (average, $0.44 \mathrm{~mm}$; SD, $0.36 \mathrm{~mm}$ ). The highest RMS was reached by the registration between the first VECTRA H1 scans and the first model obtained with the VECTRA M3 (average, $0.52 \mathrm{~mm}$; SD, $0.14 \mathrm{~mm}$ ). One-way ANOVA for correlated samples verified a statistically significant difference among the 3 groups of measurements $\left(\mathrm{F}_{2,98}=23.76\right.$; $P<.0001)$. Post hoc test highlighted statistically significant differences between M3 versus M3 and $\mathrm{H} 1$ versus
H1 and between M3 versus M3 and H1 versus M3 comparisons $(P<.001)$; in contrast, no statistically significant differences were found between $\mathrm{H} 1$ versus $\mathrm{H} 1$ and $\mathrm{H} 1$ versus $\mathrm{M} 3$ comparisons $(P=.134)$.

The same results were observed for the registration and superimposition of scans from the mannequin head. On average, RMS values from M3 versus M3 and $\mathrm{H} 1$ versus $\mathrm{H} 1$ comparisons were $0.06 \mathrm{~mm}$ (SD, $0.02 \mathrm{~mm}$ ) and $0.05 \mathrm{~mm}(\mathrm{SD}, 0.01 \mathrm{~mm})$, respectively. The RMS value from the M3 versus H1 comparison was on average $0.13 \mathrm{~mm}$ (SD, $0.01 \mathrm{~mm}$ ). One-way ANOVA highlighted statistically significant differences among the 3 groups $\left(\mathrm{F}_{2,18}=95.32 ; P<.0001\right)$. Post hoc tests verified significant differences between M3 versus $\mathrm{M} 3$ and $\mathrm{H} 1$ versus $\mathrm{M} 3$ comparisons $(P<.001)$ 
Table 4. TECHNICAL ERRORS OF MEASUREMENT FOR LINEAR DISTANCES AND ANGLES

$$
\text { M3 vs M3 }
$$

H1 vs $\mathrm{H} 1$

M3 vs H1

M3 vs H1 (Mean)

\begin{tabular}{|c|c|c|c|c|}
\hline \multicolumn{5}{|l|}{ Linear distances $(\mathrm{mm})$} \\
\hline \multicolumn{5}{|l|}{ Frontal plane } \\
\hline $\operatorname{tr}-n$ & 0.5 & 1.1 & 1.2 & 1.1 \\
\hline n-pg & 0.6 & 0.6 & 0.8 & 0.6 \\
\hline n-sn & 0.3 & 0.5 & 0.9 & 0.6 \\
\hline sn-pg & 0.4 & 0.5 & 1.0 & 0.7 \\
\hline \multicolumn{5}{|l|}{ Horizontal plane } \\
\hline$e x_{r}-e x_{1}$ & 0.7 & 0.8 & 1.9 & 1.8 \\
\hline $\mathrm{zy}_{\mathrm{r}}-\mathrm{zy}_{1}$ & 0.3 & 0.6 & 0.8 & 0.9 \\
\hline$t_{r}-t_{1}$ & 0.7 & 1.3 & 1.7 & 1.2 \\
\hline $\mathrm{ch}_{\mathrm{r}}-\mathrm{ch}_{1}$ & 0.9 & 1.2 & 1.2 & 1.1 \\
\hline $\mathrm{cph}_{\mathrm{r}}-\mathrm{cph}_{1}$ & 0.8 & 1.1 & 1.4 & 1.2 \\
\hline $\mathrm{go}_{\mathrm{r}}-\mathrm{go}_{1}$ & 0.7 & 1.1 & 1.6 & 1.6 \\
\hline \multicolumn{5}{|l|}{ Sagittal plane } \\
\hline$t_{m}-n$ & 0.9 & 0.7 & 1.2 & 0.3 \\
\hline $\mathrm{t}_{\mathrm{m}}-\mathrm{sn}$ & 0.9 & 1.0 & 1.6 & 1.4 \\
\hline$t_{m}-p g$ & 1.0 & 0.7 & 2.0 & 2.5 \\
\hline pg-go $\mathrm{m}$ & 0.5 & 1.1 & 1.2 & 1.1 \\
\hline$t_{m}-g_{m}$ & 0.9 & 0.6 & 0.9 & 1.5 \\
\hline \multicolumn{5}{|l|}{ Angles $\left(^{\circ}\right)$} \\
\hline \multicolumn{5}{|l|}{ Frontal plane } \\
\hline $\mathrm{en}_{\mathrm{r}}-\mathrm{ex}_{\mathrm{r}}$ vs TH & 1.2 & 1.0 & 1.3 & 1.6 \\
\hline $\mathrm{en}_{1}-\mathrm{ex}_{1}$ vs TH & 0.9 & 1.0 & 1.8 & 2.3 \\
\hline \multicolumn{5}{|l|}{ Horizontal plane } \\
\hline$t_{r}-n-t_{1}$ & 0.4 & 1.0 & 0.8 & 0.6 \\
\hline$t_{r}-$ prn- $t_{1}$ & 0.4 & 0.7 & 0.7 & 0.6 \\
\hline$t_{r}-p g-t_{1}$ & 0.9 & 0.5 & 0.9 & 0.7 \\
\hline go $_{\mathbf{r}}-\mathrm{pg}-\mathrm{go}_{1}$ & 0.8 & 0.8 & 1.6 & 1.7 \\
\hline \multicolumn{5}{|l|}{ Sagittal plane } \\
\hline n-sn-pg & 0.5 & 0.8 & 0.9 & 0.7 \\
\hline n-prn-pg & 0.6 & 0.4 & 0.8 & 0.5 \\
\hline sn-n-prn & 0.4 & 0.4 & 0.7 & 0.5 \\
\hline$t_{r}-g^{-}-p g$ & 1.5 & 1.1 & 1.5 & 0.9 \\
\hline $\mathrm{t}_{1}-\mathrm{go}_{1}-\mathrm{pg}$ & 1.0 & 1.1 & 1.6 & 1.6 \\
\hline$\left(t_{m}-n\right)$ vs $\left(g o_{m}-p g\right)$ & 0.8 & 1.1 & 1.7 & 1.6 \\
\hline
\end{tabular}

Abbreviations: See Tables 1 and 2.

Gibelli et al. Validation of Portable Stereophotogrammetry. J Oral Maxillofac Surg 2018.

and between $\mathrm{H} 1$ versus $\mathrm{H} 1$ and $\mathrm{H} 1$ versus $\mathrm{M} 3$ comparisons $(P<.001)$, but not between M3 versus M3 and H1 versus $\mathrm{H} 1$ comparisons $(P=.495)$.

\section{Discussion}

The recently introduced portable stereophotogrammetric devices are likely to provide an important improvement in anatomic research of the human face. ${ }^{23}$ Nevertheless, they need to be validated to assess their reliability when applied to facial imaging. ${ }^{24}$

A validation study of the VECTRA H1 system was recently performed by Camison et $\mathrm{al}^{18}$ who found that facial images obtained with the portable system were highly comparable to those obtained with the static 3dMDface system: of the 136 linear distances analyzed in their study, they found an average rTEM of $1.13 \%$ (TEM, $0.84 \mathrm{~mm}$ ). In general, errors smaller than $2 \mathrm{~mm}$ are considered appropriate for accuracy and precision in 3D photogrammetric validation,,${ }^{1,7,8}$ although 1- to 2-mm differences could be important for highly precise measurements, such as cleft lip and nasal surgery. ${ }^{25,26}$

Camison et al ${ }^{18}$ located 17 landmarks, including the traditional ones according to Farkas,${ }^{19}$ and nontraditional references to ensure adequate facial surface coverage. Because of the different protocols, the present procedure shared only a few landmarks with 
Table 5. RELATIVE TECHNICAL ERRORS OF MEASUREMENT FOR LINEAR DISTANCES AND ANGLES

$\begin{array}{llll}\text { M3 vs M3 } & \text { H1 vs H1 } & \text { M3 vs H1 } & \text { M3 vs H1 (mean) }\end{array}$

\begin{tabular}{|c|c|c|c|c|}
\hline \multicolumn{5}{|l|}{ Linear distances } \\
\hline \multicolumn{5}{|l|}{ Frontal plane } \\
\hline tr-n & $0.7^{*}$ & $1.6^{\dagger}$ & $1.8^{\dagger}$ & $1.4^{\dagger}$ \\
\hline n-pg & $0.5^{*}$ & $0.6^{*}$ & $0.7^{*}$ & $0.6^{*}$ \\
\hline n-sn & $0.5^{*}$ & $0.9^{*}$ & $1.7^{\dagger}$ & $1.1^{\dagger}$ \\
\hline sn-pg & $0.7^{*}$ & $0.9^{*}$ & $1.7^{\dagger}$ & $1.2^{\dagger}$ \\
\hline \multicolumn{5}{|l|}{ Horizontal plane } \\
\hline $\mathrm{ex}_{\mathrm{r}}-\mathrm{ex}$ & $0.8^{*}$ & $0.9^{*}$ & $2.2^{\dagger}$ & $2.0^{\dagger}$ \\
\hline $\mathrm{zy}_{\mathrm{r}}-\mathrm{zy}_{1}$ & $0.2^{*}$ & $0.4^{*}$ & $0.6^{*}$ & $0.7^{*}$ \\
\hline$t_{r}-t_{1}$ & $0.5^{*}$ & $0.9^{*}$ & $1.2^{\dagger}$ & $0.9 *$ \\
\hline $\mathrm{ch}_{\mathrm{r}}-\mathrm{ch}_{1}$ & $1.7^{\dagger}$ & $2.4^{\dagger}$ & $2.5^{\dagger}$ & $2.2^{\dagger}$ \\
\hline $\mathrm{cph}_{\mathrm{r}}-\mathrm{cph}_{1}$ & $6.7^{\ddagger}$ & $9.5^{\S}$ & $12.4^{\|}$ & 10.6 \\
\hline $\mathrm{go}_{\mathrm{r}}-\mathrm{go}_{1}$ & $0.6^{*}$ & $1.0^{\dagger}$ & $1.5^{\dagger}$ & $1.5^{\dagger}$ \\
\hline \multicolumn{5}{|l|}{ Sagittal plane } \\
\hline$t_{m}-n$ & $1.0^{\dagger}$ & $0.7^{*}$ & $1.3^{\dagger}$ & $0.4^{*}$ \\
\hline$t_{m}-s n$ & $0.9^{*}$ & $1.0^{\dagger}$ & $1.7^{\dagger}$ & $1.4^{\dagger}$ \\
\hline$t_{m}-p g$ & $0.9^{*}$ & $0.6^{*}$ & $1.3^{\dagger}$ & $1.4^{\dagger}$ \\
\hline $\mathrm{pg}-\mathrm{go}_{\mathrm{m}}$ & $0.6^{*}$ & $1.3^{\dagger}$ & $1.4^{\dagger}$ & $1.3^{\dagger}$ \\
\hline $\mathrm{t}_{\mathrm{m}}-\mathrm{go}_{\mathrm{m}}$ & $1.8^{\dagger}$ & $1.1^{\dagger}$ & $1.9^{\dagger}$ & $2.4^{\dagger}$ \\
\hline \multicolumn{5}{|l|}{ Angles } \\
\hline \multicolumn{5}{|l|}{ Frontal plane } \\
\hline $\mathrm{en}_{\mathrm{r}}-\mathrm{ex}_{\mathrm{r}}$ vs TH & $8.5^{\S}$ & $7.2^{\S}$ & $9.3^{\S}$ & 11.6 \\
\hline $\mathrm{en}_{1}-\mathrm{ex}_{1}$ vs TH & $7.1^{\ddagger}$ & $6.7^{\ddagger}$ & 10.6 & 14.9 \\
\hline \multicolumn{5}{|l|}{ Horizontal plane } \\
\hline$t_{r}-n-t_{1}$ & $0.6^{*}$ & $1.3^{\dagger}$ & $1.1^{\dagger}$ & $0.9 *$ \\
\hline$t_{r}-$ prn- $t_{1}$ & $0.7^{*}$ & $1.0^{\dagger}$ & $1.2^{\dagger}$ & $0.9 *$ \\
\hline$t_{r}-p g-t_{1}$ & $1.4^{\dagger}$ & $0.9^{*}$ & $1.5^{\dagger}$ & $1.1^{\dagger}$ \\
\hline go $_{\mathrm{r}}-\mathrm{pg}_{-} \mathrm{go}_{1}$ & $1.2^{\dagger}$ & $1.2^{\dagger}$ & $1.8^{\dagger}$ & $2.2^{\dagger}$ \\
\hline \multicolumn{5}{|l|}{ Sagittal plane } \\
\hline n-sn-pg & $0.3^{*}$ & $0.5^{*}$ & $0.5^{*}$ & $0.4^{*}$ \\
\hline n-prn-pg & $0.5^{*}$ & $0.3^{*}$ & $0.6^{*}$ & $0.4^{*}$ \\
\hline sn-n-prn & $1.7^{\dagger}$ & $1.9^{\dagger}$ & $3.1^{\dagger}$ & $2.6^{\dagger}$ \\
\hline$t_{r}-g o_{r}-p g$ & $1.3^{\dagger}$ & $0.9^{*}$ & $1.3^{\dagger}$ & $0.7^{*}$ \\
\hline$t_{1}-g_{1}-p g$ & $0.9^{*}$ & $0.9^{*}$ & $1.4^{\dagger}$ & $1.3^{\dagger}$ \\
\hline$\left(t_{m}-n\right)$ vs $\left(g_{m}-p g\right)$ & $0.7^{*}$ & $0.9^{*}$ & $1.4^{\dagger}$ & $1.4^{\dagger}$ \\
\hline
\end{tabular}

Note: All values are percentages.

Abbreviations: See Tables 1 and 2.

${ }^{*}$ Excellent relative technical error of measurement values (according to Camison et $\mathrm{al}^{18}$ ).

$\dagger$ Very good relative technical error of measurement values (according to Camison et $\mathrm{al}^{18}$ ).

$\ddagger$ Good relative technical error of measurement values (according to Camison et $\mathrm{al}^{18}$ ).

$\S$ Moderate relative technical error of measurement values (according to Camison et $\mathrm{al}^{18}$ ).

\| Poor relative technical error of measurement values (according to Camison et $\mathrm{al}^{18}$ ).

Gibelli et al. Validation of Portable Stereophotogrammetry. J Oral Maxillofac Surg 2018.

the procedure used by Camison et al, ${ }^{18}$ with differences in the type of linear distances assessed for validation. However, the present results generally confirm those of the previous study. Camison et $\mathrm{al}^{18}$ ranked the prevalence of measurements as very good (rTEM, 1.0 to $3.9 \%$ ), as did the present investigation. The present average TEMs for the intra-device comparisons were $1.29 \mathrm{~mm}$ and $1.19^{\circ}\left(1.17 \mathrm{~mm}\right.$ and $1.11^{\circ}$ if the mean $\mathrm{H} 1$ and $\mathrm{M} 3$ values are used). From a clinical point of view, these differences appear negligible for most practical applications and probably unappreciable by most observers.

Only 3 measurements showed low repeatability: cph-cph distance and inclinations of the palpebral fissures versus the true horizontal plane. The discordance of these 3 measurements compared with the other measurements has 2 possible explanations. First, there is the effect of facial mimicry (slight labial and eye movements), as reported in the literature, ${ }^{27,28}$ especially for the lower part of the face. ${ }^{29}$ Second, 


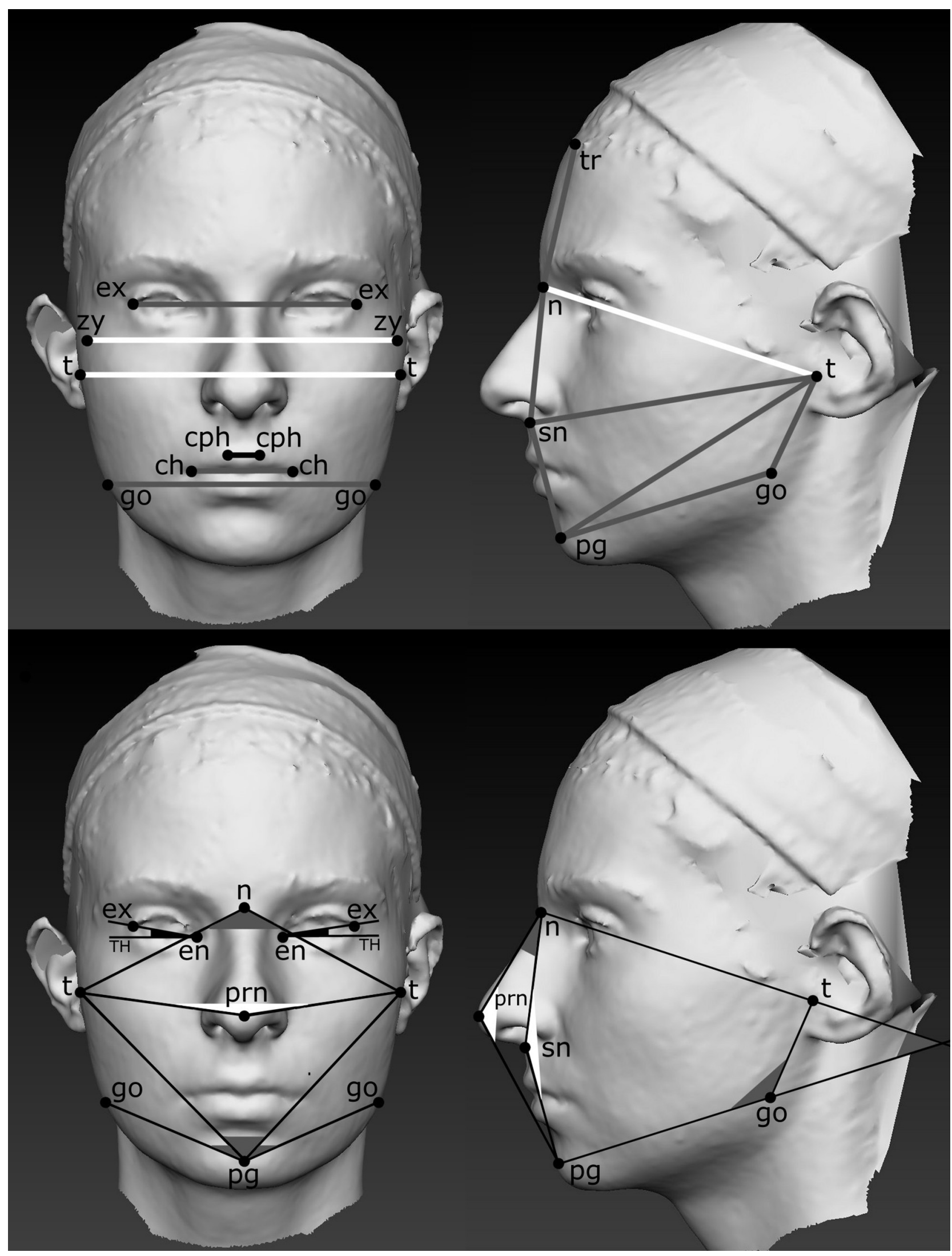

FIGURE 3. Linear distances and angles analyzed in the present study and their repeatability according to the Bland-Altman test for the VECTRA $M 3$ versus $\mathrm{H} 1$ device (white lines and angles, repeatability $\geq 95 \%$; gray lines and angles, repeatability 85.1 to $94.9 \%$; black lines and angles, repeatability $\leq 85 \%)$. ch, cheilion; cph, crista philtri; en, endocanthion; ex, exocanthion; go, gonion; n, nasion; pg, pogonion; prn, pronasale; sn, subnasale; $t$, tragus; tr, trichion; zy, zygion. 
Table 6. REPEATABILITY ACCORDING TO BA, TEM, AND RTEM FOR FAI SURFACE AREA AND VOLUME

\begin{tabular}{lcccc} 
FAI & M3 vs M3 & H1 vs H1 & M3 vs H1 & M3 vs H1 (Mean) \\
\hline Surface area & & & & \\
BA (\%) & 95.2 & 95.4 & 93.1 & 4.8 \\
TEM (cm $\left.{ }^{2}\right)$ & 2.7 & 2.6 & 1.4 & 1.3 \\
rTEM (\%) & 0.79 & 0.76 & 61.5 & 74.5 \\
Volume & & & 53.6 & 50.9 \\
BA (\%) & 87.6 & 54.2 & 7.7 & 6.2 \\
TEM (cm $\left.{ }^{3}\right)$ & 17.3 & 67.4 & 8.0 & \\
rTEM (\%) & 2.2 & & & \\
\hline
\end{tabular}

Abbreviations: BA, Bland-Altman test; FAI, facial area of interest; rTEM, relative technical error of measurements; TEM, absolute technical error of measurements.

Gibelli et al. Validation of Portable Stereophotogrammetry. J Oral Maxillofac Surg 2018.

these 3 measurements had the least magnitude, and previous studies have reported that reliability decreases as measurements decrease, specifically philtrum breadth. ${ }^{30,31}$

In all cases, the use of $\mathrm{H} 1$ scans worsened TEM and rTEM values in $\mathrm{H} 1$ versus $\mathrm{H} 1$ and $\mathrm{M} 3$ versus $\mathrm{H} 1$ comparisons, probably because of the influence of involuntary facial movements. For the latter case, the assessment of mean values between 2 consecutive H1 scans seemed to improve repeatability and TEM and rTEM values and could be used to minimize the effects of subtle facial changes during acquisition, although this amelioration is slight and does not lead to an improvement of rTEM in most cases.

Another type of measurement of great interest in 3D facial imaging is the RMS point-to-point distance between 2 3D facial surfaces: facial registration and superimposition offer an innovative representation of the modifications from surgical procedures or facial mimicry, with a number of applications in different fields. $^{14,16,32}$ Therefore, the validation of novel portable stereophotogrammetric devices also should consider the application of these procedures.

For the RMS point-to-point distance, Camison et $\mathrm{al}^{18}$ found a mean value of $0.43 \mathrm{~mm}$ in the comparison between the VECTRA H1 and 3dMD systems, similar to the comparison between the VECTRA H1 and M3 devices in the present study. In addition, they found a mean RMS value of $0.034 \mathrm{~mm}$ for the comparison of mannequin head scans obtained with the VECTRA $\mathrm{H} 1$ and a global RMS value of $0.14 \mathrm{~mm}$ when the 2 registered mannequin head models were obtained with the $\mathrm{H} 1$ and $3 \mathrm{dMD}$ systems. From these results, they stated that one can expect consistent results from scan to scan using the same VECTRA H1 device. ${ }^{18}$ The present data confirm the results by Camison et $\mathrm{al}^{18}$ and provide an additional comparison with RMS values obtained through the registration of 2 scans from the static M3 device. Results confirmed the consistent impact of involuntary facial move- ments, proved by the higher RMS values for $\mathrm{H} 1$ versus H1 facial model registration than for M3 versus M3 superimposition. Moreover, the superimposition of 2 models acquired through different devices showed a higher RMS value in mannequin head models that did not include movements. In contrast, $\mathrm{H} 1$ versus H1 and M3 versus M3 comparisons did not notably vary in mannequin head acquisitions, confirming the influence of facial movements when the portable device is used.

Therefore, the present study highlights an important caveat for the application of portable stereophotogrammetric devices for 3D-to-3D surface registration and superimposition. In addition, it cautions against the application of registration procedures to scans acquired through portable and static devices, because the metric parameters for assessing point-to-point distances are expected to increase.

The present study adds new additional information to the previous study. ${ }^{18}$ First, the comparison was performed between devices produced by the same company, limiting the possible influence of machines and software produced by different companies. The static VECTRA M3 was considered the reference and has been validated in the literature. ${ }^{6,32}$ Moreover, the present data on $\mathrm{M} 3$ versus $\mathrm{M} 3$ registration and superimposition confirmed the previous validation study of the static device. ${ }^{6}$

Second, the comparison between the VECTRA H1 and M3 was performed after an intra-device validation. This step is crucial to assess the repeatability of all measurements applied in the comparison between scans obtained through the portable device.

Third, the measurement protocol was standardized to decrease possible influences from the operator. For example, the selection of the FAI was semiautomatically performed according to anatomic landmarks previously marked on the volunteers' faces, whereas Camison et $\mathrm{al}^{18}$ manually selected the facial area. 

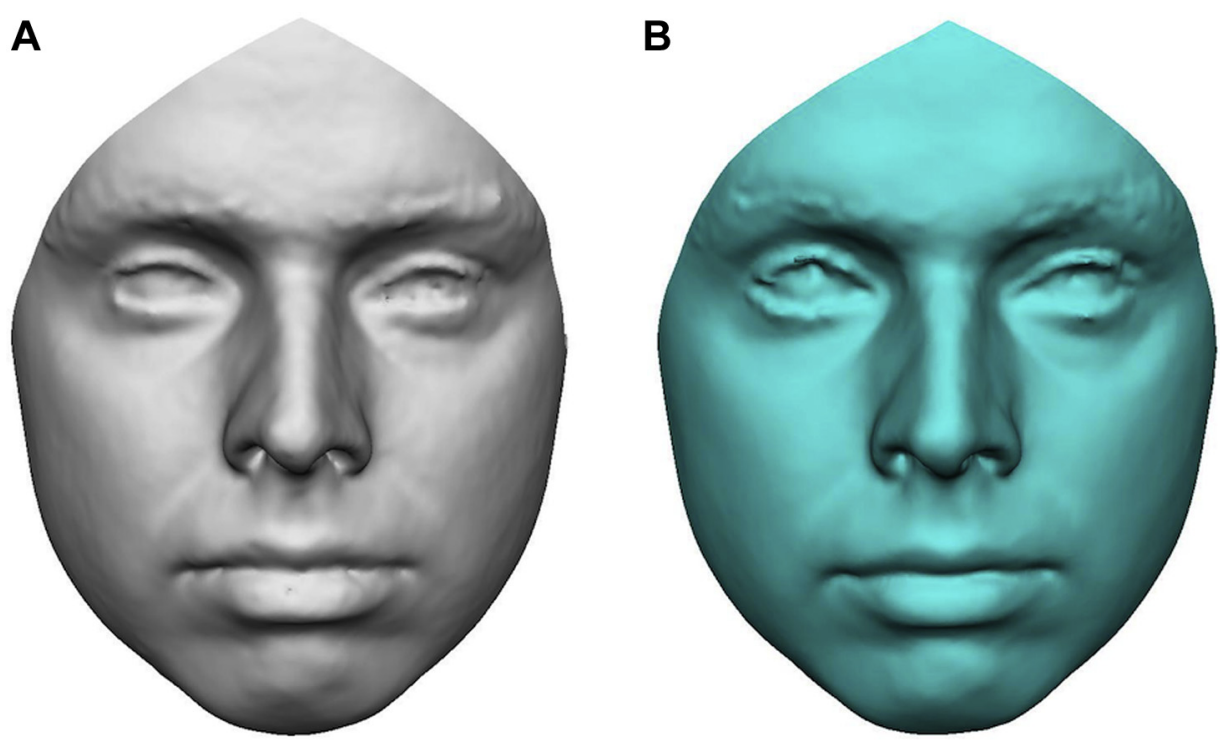

C

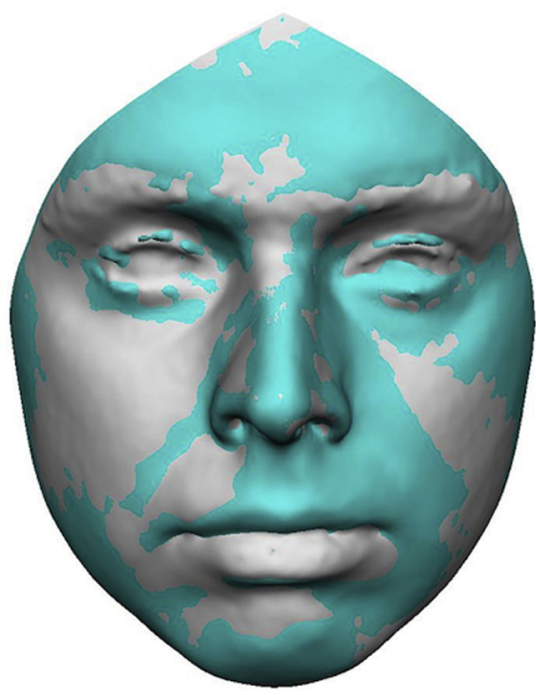

D

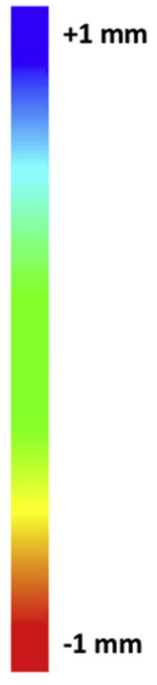

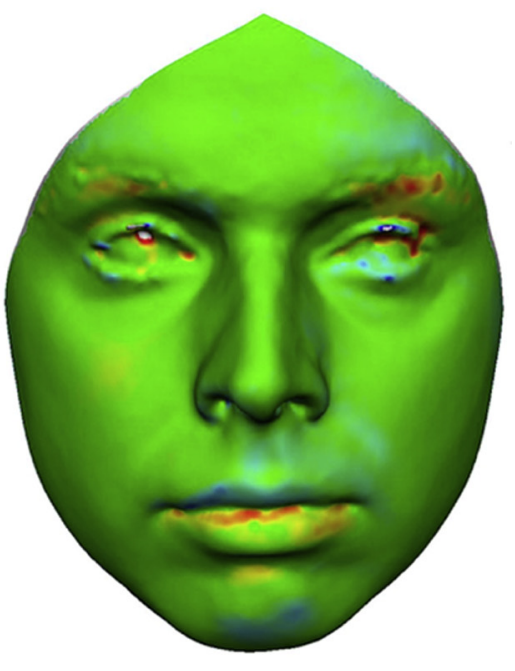

FIGURE 4. Example of registration, superimposition, and assessment of point-to-point distances between 2 facial surfaces. $A$, First scan from the VECTRA HI device. $B$, Second scan from the VECTRA HI device of the same subject. $C$, Registration of the 2 3-dimensional surfaces according to the shortest point-to-point distance. $D$, Elaboration of a chromatic facial analysis with different colors (green, superimposed areas; red and blue, discordant areas between the 2 scans). The subject is one of the authors.

Gibelli et al. Validation of Portable Stereophotogrammetry. J Oral Maxillofac Surg 2018.

Fourth, the present validation study was performed to explore not only linear distances, angles, and RMS values but also surface area and volume measurements, thus providing a more complete analysis of the performance of the VECTRA H1. Surface areas and volumes are novel parameters that can be easily assessed on 3D facial models compared with traditional cephalometry based on the classic linear distances and angles and are gaining greater importance in the literature. $^{4,5,17,33-36}$

For what concerns these measurements, the present results add an important contribution for the validation of the VECTRA H1 device, because surface areas could be reliably assessed. In contrast, repeatability of volumes decreased with the portable device compared with the static device, with the lowest performance for $\mathrm{H} 1$ versus M3 scans. This result could be due to the strong impact of involuntary facial and head movements during the acquisition procedure using the $\mathrm{H} 1$ device, because it requires 3 consecutive captured images, whereas the static M3 device captures the same images simultaneously. As a consequence, even subtle changes in facial morphology can lower the repeatability of all measurements, although only facial volume showed a decrease in repeatability below acceptable limits for facial 
imaging. In fact, for all measurements considered in the present study, repeatability and TEM and rTEM values were highest for the $\mathrm{M} 3$ versus $\mathrm{M} 3$ comparison, lower for the $\mathrm{H} 1$ versus $\mathrm{H} 1$ comparison, and lowest for the $\mathrm{H} 1$ versus $\mathrm{M} 3$ comparison.

An important limitation of the present investigation is the participation of only collaborative adults. The comparison between data collected by the 2 devices could differ for children and uncooperative persons who might move their head and face more. ${ }^{10,11}$ Furthermore, all data collection was performed in a research laboratory. Other indoor and outdoor locations can introduce environmental noise, worsening the quality of facial scans.

All acquisitions were made after labeling the landmarks of interest on each face according to the authors' standardized procedure. ${ }^{6}$ Previous studies have found that marking landmarks before taking measurements increases precision, regardless of method. ${ }^{7}$ The present reproducibility could have decreased if facial scans had been obtained without prior landmark labeling.

In conclusion, the present study provides an important contribution to the validation of the novel portable stereophotogrammetric devices. The handheld VECTRA H1 system proved reliable in assessing linear, angular, and surface area measurements, whereas volume assessment and 3D-to-3D registration were affected by unavoidable facial movements between consecutive captures. In addition, caution should be used for 3D-to-3D registration of scans from portable and static devices. These results will assist in the validation of innovative 3D acquisition systems in facial anatomy and imaging.

In addition to the validity and repeatability of measurements, there are other technical aspects to be considered. The portable instrument does not need a space to be housed, and it can be used outside the laboratory to meet patients and subjects where they live and work. In addition, its cost is approximately half that of the static system. In addition to lower repeatability compared with the fixed instrument, it takes longer to acquire the final 3D facial model, and an immediate simulation cannot be performed. When choosing an instrument for data collection, the advantages and limitations should be carefully considered, and the choice should be governed by the final goal of each investigation.

\section{Acknowledgment}

The research was performed thanks to a free and unconditional temporary loan of the VECTRA H1 system by Canfield Scientific, Inc (Fairfield, NJ). The authors are grateful to Dr Francesco Colella for the loan and to all the volunteers for their participation.

\section{References}

1. Wong JY, Oh AK, Ohta E, et al: Validity and reliability of craniofacial anthropometric measurement of 3D digital photogrammetric images. Cleft Palate Craniofac J 45:232, 2008

2. Hong C, Choi K, Kachroo Y, et al: Evaluation of the 3dMDface system as a tool for soft tissue analysis. Orthod Craniofac Res 20(suppl 1):119, 2017

3. Sawyer AR, See M, Nduka C: 3D stereophotogrammetry quantitative lip analysis. Aesth Plast Surg 33:497, 2009

4. Gibelli D, Codari M, Rosati R, et al: A quantitative analysis of lip aesthetics: The influence of gender and aging. Aesth Plast Surg 39:771, 2015

5. Codari M, Pucciarelli V, Pisoni L, et al: Laser scanner compared with stereophotogrammetry for measurements of area on nasal plaster casts. Br J Oral Maxillofac Surg 53:769, 2015

6. De Menezes M, Rosati R, Ferrario VF, et al: Accuracy and reproducibility of a 3-dimensional stereophotogrammetric imaging system. J Oral Maxillofac Surg 68:2129, 2010

7. Weinberg SM, Scott NM, Neiswanger K, et al: Digital threedimensional photogrammetry: Evaluation of anthropometric precision and accuracy using a Genex 3D camera system. Cleft Palate Craniofac J 41:507, 2004

8. Weinberg SM, Naidoo S, Govier DP, et al: Anthropometric precision and accuracy of digital three-dimensional photogrammetry: Comparing the Genex and 3dMD imaging systems with one another and with direct anthropometry. J Craniofac Surg 17: 477,2006

9. Koudelovà J, Bruzek J, Cagànovà V, et al: Development of facial sexual dimorphism in children aged between 12 and 15 years: A three-dimensional longitudinal study. Orthod Craniofac Res $18: 175,2015$

10. Pucciarelli V, Bertoli S, Codari M, et al: Facial evaluation in holoprosencephaly. J Craniofac Surg 28:e22, 2017

11. Pucciarelli V, Bertoli S, Codari M, et al: The face of Glut1-DS patients: A 3D craniofacial morphometric analysis. Clin Anat 30:644, 2017

12. Othman SA, Ahmad R, Asi SM, et al: Three-dimensional quantitative evaluation of facial morphology in adults with unilateral cleft lip and palate, and patients without clefts. Br J Oral Maxillofac Surg 52:208, 2014

13. Darby LJ, Millett DT, Kelly N, et al: The effect of smiling on facial asymmetry in adults: a 3D evaluation. Aust Orthod J31:132, 2015

14. Gibelli D, De Angelis D, Poppa P, et al: An assessment of how facial mimicry can change facial morphology: implications for identification. J Forensic Sci 62:405, 2017

15. Sawyer AR, See M, Nduka C: Quantitative analysis of normal smile with 3D stereophotogrammetry - An aid to facial reanimation. J Plast Reconstr Aesthet Surg 63:65, 2010

16. Gibelli D, Codari M, Pucciarelli V, et al: A quantitative assessment of lips movements in different facial expressions through 3D-3D superimposition: A cross-sectional study [published online ahead of print November 23, 2017]. J Oral Maxillofac Surg https://doi.org/10.1016/j.joms.2017.11.017

17. Tzou CH, Artner NM, Pona I, et al: Comparison of threedimensional surface-imaging systems. J Plast Reconstr Aesthet Surg 67:489, 2014

18. Camison L, Bykowski M, Lee WW, et al: Validation of the Vectra H1 portable three-dimensional photogrammetry system for facial imaging. Int J Oral Maxillofac Surg 47:403, 2018 https:// doi.org/10.1016/j.ijom.2017.08.008

19. Farkas LG: Anthropometry of the Head and Face. New York, NY, Raven Press, 1994

20. Codari M, Pucciarelli V, Stangoni F, et al: Facial thirds-based evaluation of facial asymmetry using stereophotogrammetric devices: Application to facial palsy subjects. J Craniomaxillofac Surg 45:76, 2017

21. Giavarina D: Understanding Bland Altman analysis. Biochem Med 25:141, 2015

22. Adao Perini T, Lameira de Oliveira G, dos Santos Ornellas J, et al: Technical error of measurement in anthropometry. Rev Bras Med Esporte 11:86, 2005 
23. Ceinos R, Tardivo D, Bertrand MF, et al: Inter-and intra-operator reliability of facial and dental measurements using 3D-stereophotogrammetry. J Esthet Restor Dent 28:178, 2016

24. Lubbers HT, Medinger L, Kruse AL, et al: The influence of involuntary facial movements on craniofacial anthropometry: A survey using a three-dimensional photographic system. Br J Oral Maxillofac Surg 50:171, 2012

25. Maal TJ, van Loon B, Plooij JM, et al: Registration of 3dimensional facial photographs for clinical use. J Oral Maxillofac Surg 68:2391, 2010

26. Van Loon B, Maal TJ, Plooij JM, et al: 3D stereophotogrammetric assessment of pre- and post-operative volumetric changes in the cleft lip and palate nose. Int J Oral Maxillofac Surg 39:534, 2010

27. Ferrario VF, Sforza C, Poggio CE, et al: Preliminary evaluation of an electromagnetic three-dimensional digitizer in facial anthropometry. Cleft Palate Craniofac J 35:9, 1998

28. de Menezes M, Rosati R, Allevi C, et al: A photographic system for the three-dimensional study of facial morphology. Angle Orthod 79:1070, 2009

29. Maal TJJ, van Loon B, Plooij JM, et al: Variation of the face in rest using 3D stereophotogrammetry. Int J Oral Maxillofac Surg 40: 1252,2011
30. Ward RE, Jamison PL: Measurement precision and reliability in craniofacial anthropometry: Implications and suggestions for clinical applications. J Craniofac Genet Dev Biol 11:156, 1991

31. Jamison PL, Ward RE: Brief communication: Measurement size, precision, and reliability in craniofacial anthropometry: bigger is better. Am J Phys Anthropol 90:495, 1993

32. Gibelli D, De Angelis D, Poppa P, et al: A view to the future: A novel approach for 3D-3D superimposition and quantification of differences from next-generation video surveillance systems. J Forensic Sci 62:457, 2017

33. Hall RL: Energetics of nose and mouth breathing, body size, body composition, and nose volume in young adult males and females. Am J Hum Biol 17:321, 2005

34. Heidari H, Mahmoudzadeh-Sagheb H, Khammar T, et al: Anthropometric measurements of the external nose in 18-25-year-old Sistani and Baluch aborigine women in the South-East of Iran. Folia Morphol 68:88, 2009

35. Yoo JH, Lee YH, Lee H, et al: Correlation between orbital volume, body mass index, and eyeball position in healthy East Asians. J Craniofac Surg 24:822, 2013

36. Ozer CM, Oz II, Serifoglu I, et al: Evaluation of eyeball and orbit in relation to gender and age. J Craniofac Surg 27:e793, 2016 